\title{
The AMANDA-II Neutrino Telescope
}

\author{
R. Wischnewski ${ }^{\text {a }}$ for the AMANDA-Collaboration

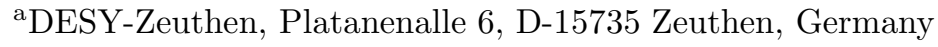

The AMANDA-II telescope at the South Pole is constructed of 677 optical modules at 19 strings. We describe the observation of atmospheric neutrinos with the first stage 10-string detector AMANDA-B10, which establishes AMANDA as a working neutrino telescope. The expected performance for the AMANDA-II detector is discussed.

\section{INTRODUCTION}

To detect the feeble flux of predicted high energy astrophysical neutrinos, high energy Cherenkov neutrino telescopes are build as a grid of photomultiplier tubes (PMTs) covering a huge geometric volume. The density of PMTs per effective detector area is about 3 orders of magnitude below that for $\nu$-events triggered in the SuperKamiokande detector; this implies a detailed verification of the anticipated performance of these new detectors.

The AMANDA-II telescope is located at the geographic South Pole and uses the transparent ice of the $3 \mathrm{~km}$ thick ice sheet [1]. The detector has been installed between November 1995 and February 2000, and consists of 677 PMTs in optical modules $(\mathrm{OM})$ on 19 vertical strings deployed to depths between $1300 \mathrm{~m}-2400 \mathrm{~m}$. The main instrumented volume ranges from $1500 \mathrm{~m}-2000 \mathrm{~m}$, it covers a cylinder of $200 \mathrm{~m}$ diameter (fig.11).

Strings 1-10 form the inner detector of $120 \mathrm{~m}$ diameter called AMANDA-B10, which was commissioned in 1997. They use passive OMs with electrical analog signal transmission over $2 \mathrm{~km}$ cable. Strings 11-19 form the outer cylinder and are based on analog fiber transmission [2].

First physics results obtained with AMANDAB10 are presented at this conference by [3].

\section{2. $\nu_{\mu}$-SEPARATION IN AMANDA-B10}

With no astrophysical neutrino sources detected yet, atmospheric neutrinos are the only source to calibrate the sensitivity to the favoured

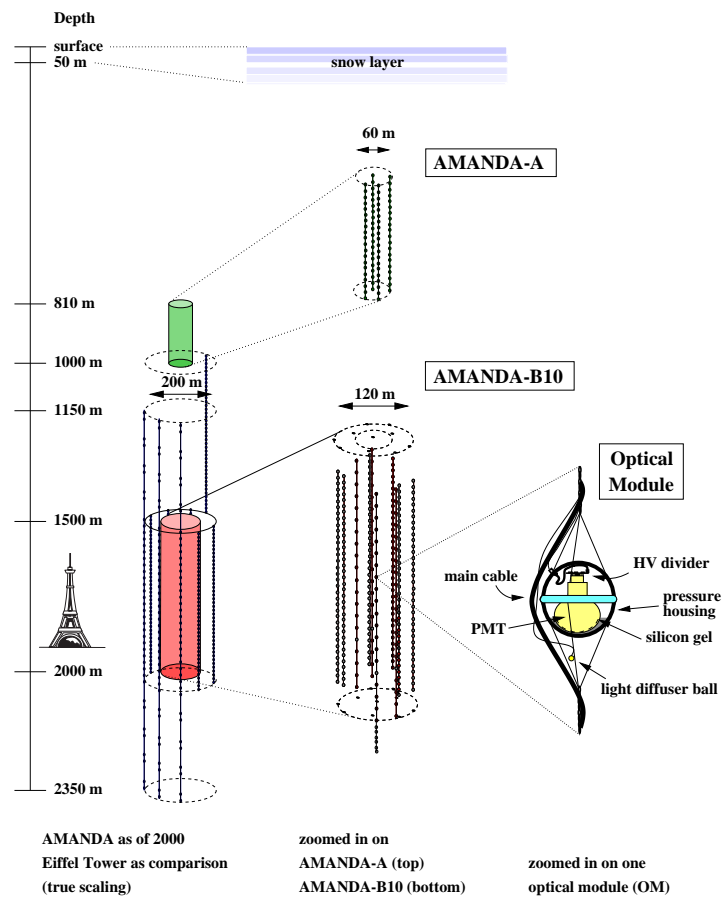

Figure 1. The AMANDA-II detector.

$\nu$-channel: upgoing $\mu$ 's generated in charged current $(\mathrm{CC}) \nu_{\mu}$-interactions.

Muon track reconstruction is based on a maximum likelidood fit of measured photon arrival times to expected arrival time distributions 昍河, and uses modeled optical bulk ice properties (light scattering and absorption), and local hole ice effects. Light emitted by muon-induced electromagnetic showers and accompanying muons (not in the track fit model) and the low informa- 


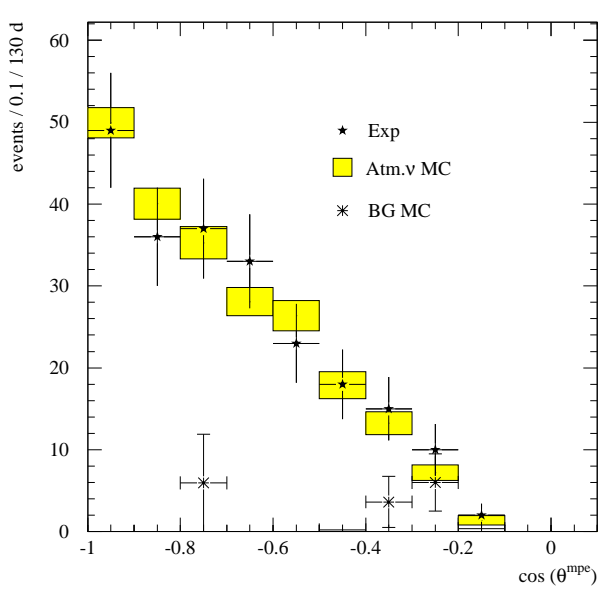

Figure 2. Zenith angle distribution for a selected neutrino sample of 223 events. Hatched boxes are $\mathrm{MC}$ expectation for atmospheric neutrinos.

tion density per track result in downgoing atmospheric muons being sometimes misreconstructed as upward tracks. They form the dominant background to $\nu_{\mu}$ 's, since they trigger about $10^{5}$ more abundant.

The 1997 data set of the AMANDA-B10 detector has been analyzed by two independent groups. They used different modifications of track reconstruction and extended cuts for selecting highest quality upward reconstructed muon tracks to reject the background at acceptable signal efficiencies. From a total sample of $10^{9}$ events triggered during 130 effective livetime days in 1997, final samples of 223 and 204 events have been extracted, with $\nu_{\mu}$ passing rates of $3-4 \%$ and residual background contamination of $10 \%$, 4 [5]. The observed number of events is consistent with expectation, within a $\sim 50 \%$ systematic error due to ice property and primary flux uncertainties. In figure 2 the distribution in cosine of the zenith angle after final cuts for the sample of analysis A is shown (compared to $\nu$-simulation expectation, normalized to experimental data). Angular acceptance of AMANDA-B10 is seen to be worst for horizontal directions. The angular and energy averaged effective muon area for muon energies of $0.1-1 \mathrm{TeV}$ is $2800 \mathrm{~m}^{2}$ (weighted over the atmospheric $\nu$-spectrum). Much higher effective areas

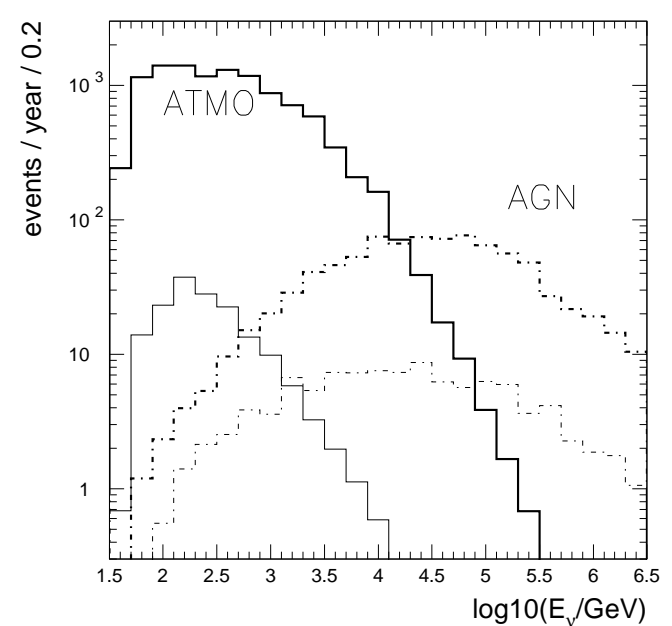

Figure 3. Neutrino energy spectrum of events, triggering AMANDA-II. Shown are charged current $\nu_{\mu}$ events (thick line) and charged current $\nu_{e}$ events (thin line) for atmospheric neutrinos (full line) and an AGN like spectrum (dashed line). Event rate is calculated per livetime year.

are observed for other searches (see below).

\section{PERFORMANCE OF AMANDA-II}

The AMANDA-II telescope is in stable operation since its commissioning in February, 2000. Reasonable agreement is found between data and simulation [2].

For neutrinos from astrophysical sources (such as Active Galactic Nuclei, AGN), harder spectra than for atmospheric neutrinos are expected. Fig. 3 shows the expected spectrum of triggered events for atmospheric neutrinos (full line) and for a spectrum of AGN neutrinos (dashed line, equal flux set for $\nu_{\mu}$ and $\nu_{e}$ ) with an assumed diffuse flux of $10^{-6} \cdot E^{-2} \mathrm{GeV} \mathrm{sr}^{-1} \mathrm{~s}^{-1} \mathrm{~cm}^{-2}$. Shown are $\mathrm{CC} \nu_{\mu}$ and $\mathrm{CC} \nu_{e}$ reactions. The total number of triggered upgoing events per livetime year for the different channels are given in table 1, including neutral current (NC) interactions.

The $\nu_{e}$-channel was recently shown to yield good sensitivity for diffuse high energy $\nu$-search [6][7. A first detection of the atmospheric neutrino induced cascades is an interesting challenge in view of the low event rate, see [7]. 


\begin{tabular}{|c|c|c|}
\hline & Atmospheric & AGN \\
\hline$\nu_{\mu}$ & $11000(\mathrm{CC}), 130(\mathrm{NC})$ & $853(\mathrm{CC})$ \\
$\nu_{e}$ & $162(\mathrm{CC}), 8.8(\mathrm{NC})$ & $103(\mathrm{CC})$ \\
\hline
\end{tabular}

Table 1

Triggered $\nu$-events per year in AMANDA-II.

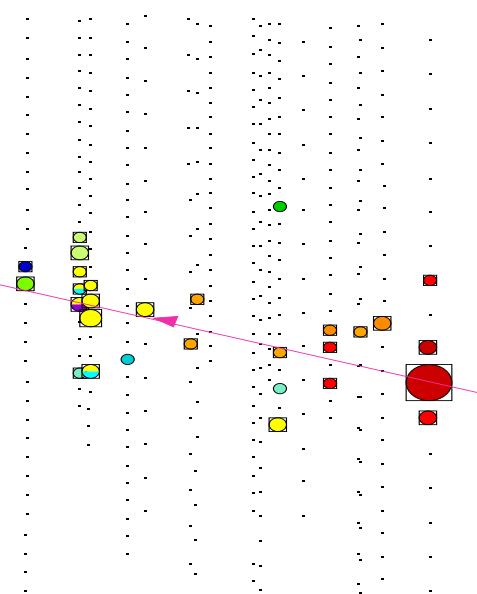

Figure 4. An upward reconstructed AMANDA-II event close to the horizon. Shown is the central part of the detector, colorscale and symbolsize correspond to hit time and amplitude.

The analysis of AMANDA-II data from 2000 is under way. With $\nu_{\mu}$-selection cuts tailored to the larger detector, we find the angular acceptance for horizontal directions and the total signal passing rate much improved, compared to AMANDAB10. Fig.t displays a good candidate event for an upward moving muon, reconstructed close to the horizon (zenith angle $=105^{\circ}$ ).

In figure 5 , the calculated AMANDA-II effective area for $10 \mathrm{TeV}$ muons is shown as function of the zenith angle at trigger level [8]. Expected effective areas for two high energy physics analyses are also shown: GRB and point source cuts, yielding areas of $30-60.000 \mathrm{~m}^{2}$. The curve shown for the AMANDA-B10 reveals the acceptance improvement in horizontal direction.

\section{CONCLUSION}

The AMANDA-II telescope reaches effective muon detection areas at $10 \mathrm{TeV}$ of up

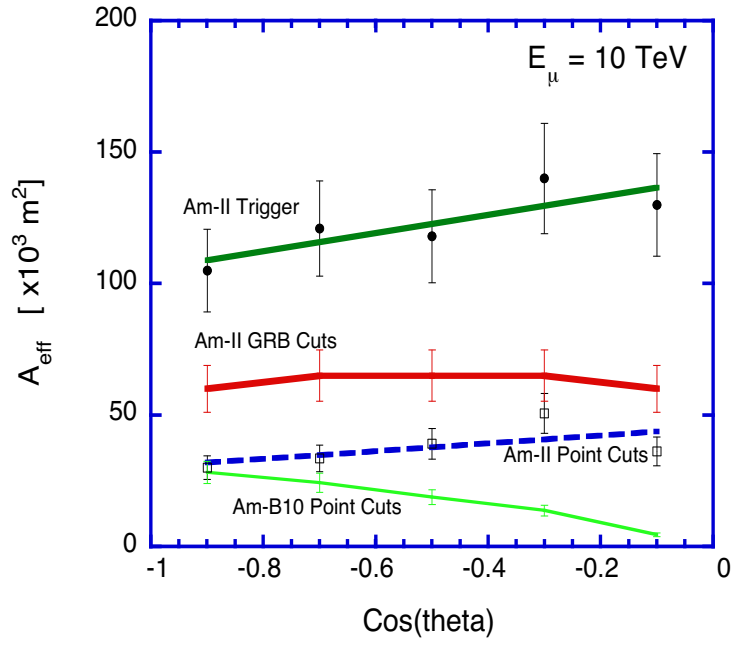

Figure 5. Effective area versus zenith angle for AMANDA-II for muons at $10 \mathrm{TeV}$, at trigger level and for GRB and point source analysis.

to $50000 \mathrm{~m}^{2}$, depending on the physics objective, and offers better angular coverage than AMANDA-B10. The latter was proven to work as a high energy neutrino detector by the isolation of a combined sample of 325 atmospheric $\nu_{\mu}$ 's. The rate of atmospheric $\nu_{\mu}$ events for the full AMANDA-II detector will be about 3 times higher, yielding 800-1000 $\nu_{\mu}$ 's for year 2000. Flux limits obtainable by AMANDA-II for high energy neutrinos from diffuse and point sources will improve by $\sim 10$ times within 2 years of livetime $[8]$.

\section{REFERENCES}

1. Andres, E., et al., Nature 410, 441, 2001.

2. R. Wischnewski, et al., Proc. $27^{\text {th }}$ ICRC, Hamburg, 1105 (2001).

3. A. Hallgren, these proceedings.

4. Ch. Wiebusch, et al., Proc. $27^{\text {th }}$ ICRC, Hamburg, 1109 (2001).

5. Ahrens, J., et al., Observation of High Energy Atmospheric Neutrinos with AMANDA, subm. to Phys. Rev. D

6. G.V. Domogatski, these proceedings.

7. Tabaoda, I., Kowalski, M. et al., Proc. $27^{\text {th }}$ ICRC, Hamburg, 1109 (2001).

8. S. Barwick, et al., ibid, p.1101. 